\title{
YAP-Dependent Induction of CD47-Enriched Extracellular Vesicles Inhibits Dendritic Cell Activation and Ameliorates Hepatic Ischemia-Reperfusion Injury
}

\author{
Zenan Yuan, ${ }^{1,2}$ Linsen Ye, ${ }^{1,2}$ Xiao Feng, ${ }^{1,2}$ Tian Zhou, ${ }^{3}$ Yi Zhou, ${ }^{4}$ Shuguang Zhu, ${ }^{1,2}$ \\ Changchang Jia, ${ }^{5}$ Haibo Li, ${ }^{1,2}$ Dongbo Qiu, ${ }^{5}$ Kun Li, ${ }^{1,2}$ Wei Liu, ${ }^{1,2}$ Yang Li, ${ }^{1,2}$ Hui Tang, \\ Guoying Wang, ${ }^{1,2}$ Qi Zhang, 5 Yang Yang $\mathbb{D}^{1,2}$ Guihua Chen $\mathbb{D}^{1,2}$ and Hua Li $\mathbb{D}^{1,2}$ \\ ${ }^{1}$ Department of Hepatic Surgery and Liver Transplantation Center, The Third Affiliated Hospital of Sun Yat-sen University, \\ Guangzhou, China \\ ${ }^{2}$ Guangdong Provincial Key Laboratory of Liver Disease Research, Guangzhou, China \\ ${ }^{3}$ State Key Laboratory of Ophthalmology, Zhongshan Ophthalmic Center, Sun Yat-sen University, Guangzhou, China \\ ${ }^{4}$ Department of General Surgery, Guangdong No.2 Provincial People's Hospital, Guangdong Province, China \\ ${ }^{5}$ Cell-Gene Therapy Translational Medicine Research Center, The Third Affiliated Hospital of Sun Yat-sen University, \\ Guangzhou, China
}

Correspondence should be addressed to Yang Yang; yyang1971@126.com, Guihua Chen; chgh1955@126.com, and Hua Li; lihua3@mail.sysu.edu.cn

Received 17 December 2020; Revised 10 May 2021; Accepted 30 May 2021; Published 22 June 2021

Academic Editor: Olga Pechanova

Copyright (C) 2021 Zenan Yuan et al. This is an open access article distributed under the Creative Commons Attribution License, which permits unrestricted use, distribution, and reproduction in any medium, provided the original work is properly cited.

\begin{abstract}
Hepatic ischemia-reperfusion injury (IRI) is the most common cause of liver damage leading to surgical failures in hepatectomy and liver transplantation. Extensive inflammatory reactions and oxidative responses are reported to be the major processes exacerbating IRI. The involvement of Yes-associated protein (YAP) in either process has been suggested, but the role and mechanism of YAP in IRI remain unclear. In this study, we constructed hepatocyte-specific YAP knockout (YAP-HKO) mice and induced a hepatic IRI model. Surprisingly, the amount of serum EVs decreased in YAP-HKO compared to WT mice during hepatic IRI. Then, we found that the activation of YAP increased EV secretion through F-actin by increasing membrane formation, while inhibiting the fusion of multivesicular body (MVB) and lysosomes in hepatocytes. Further, to explore the essential elements of YAP-induced EVs, we applied mass spectrometry and noticed CD47 was among the top targets highly expressed on hepatocyte-derived EVs. Thus, we enriched CD $47^{+}$EVs by microbeads and applied the isolated CD47 $7^{+} \mathrm{EVs}$ on IRI mice. We found ameliorated IRI symptoms after CD $47^{+} \mathrm{EV}$ treatment in these mice, and CD $47^{+} \mathrm{EVs}$ bound to CD172 $\alpha$ on the surface of dendritic cells (DCs), which inhibited DC activation and the cascade of inflammatory responses. Our data showed that CD47-enriched EVs were released in a YAP-dependent manner by hepatocytes, which could inhibit DC activation and contribute to the amelioration of hepatic IRI. CD $47^{+}$EVs could be a potential strategy for treating hepatic IRI.
\end{abstract}

\section{Introduction}

Hepatic IRI occurs when blood flow is restored after a period of hepatic ischemia $[1,2]$. Since the donated grafts are often highly susceptible to IRI, while the available organs are in severe shortage, IRI has become one of the main obstacles in liver transplantation [3]. There is an urgent need for devel- oping protective strategies against IRI to promote the survival of patients after liver transplantation.

The mechanisms governing IRI are highly complex and have been the focus of investigation for decades with numerous factors been identified with specific function. Among the principle factors in hepatic IRI, Yes-associated protein (YAP), the key effector of the Hippo pathway, has been 
reported to be a hinge joint in inflammation and oxidative stress, but its role and mechanism remain unclear. Some reports suggested that YAP played a protective role in the hepatic IRI model [4], while others found that YAPexpressing hepatocytes activated inflammation and aggregated liver fibrosis through the YAP/TAZ/CYR61 axis [5]. These conflicting findings suggested that the mechanisms in YAP's function have not been fully unveiled, since YAP has been found to regulate the binding of actin and angiomotin (AMOT) family members [6], which play an irreplaceable role in endosomal transport and the secretion of extracellular vesicles (EVs) [7]. Here, we investigated the paracrine effect of YAP through the secretion of EVs.

EVs are phospholipid bilayer vesicles widely distributed in body fluids as a form of intercellular communication and modulation of cellular activities in recipient cells. Multiple immunomodulatory effects of EVs have been reported [8, 9], but the mechanism linking hepatic injury to associated immune responses through EVs has not been found. Initiated by hypoxic stress, IRI displayed extensive inflammatory responses that are driven by innate immunity and supported by adaptive immunity $[4,10]$. Hepatic DCs act as one of the major mediators in local immune responses [11]. Activated DCs could [12] trigger both innate and adaptive immunity and aggregate local injury. However, reports also showed that hepatic DCs could limit certain inflammation and promote immune tolerance [13]. The immune-modulatory activity of DCs is regulated by the expression level of CD47, the well-known "don't eat me" signal [14]. By binding to the counter-receptor signal-regulated protein alpha (SIRP alpha/CD172 $\alpha$ ), which is mainly expressed on the surface of myeloid cells, CD47 could initiate the inhibitory signaling to restrain inflammation $[15,16]$.

In this study, we found CD47 on hepatic EVs targeted $\mathrm{CD}_{172}{ }^{+} \mathrm{DCs}$ and potently inhibited their activation, therefore alleviated hepatic IRI, whereas YAP expression is required for EV secretion of hepatocytes. Mechanistically, YAP induced EV secretion through F-actin by increasing membrane formation, with the inhibition of MVB and lysosome fusion. Our results revealed a novel mechanism for maintaining immune balance in hepatic IRI, via the regulation of YAP-EV-CD47 axis in hepatocyte-DC crosstalk, which suggested a novel therapeutic strategy utilizing $\mathrm{CD} 47^{+} \mathrm{EVs}$ in treating hepatic IRI.

\section{Methods}

2.1. Human Subjects. The selected samples were from 69 patients who underwent liver transplantation from donors after cardiac death (DCD) since April 2010 to April 2015 at the liver transplantation center of the third affiliated hospital, Sun Yat-sen University. Cases of 64 males and 5 females were included, with an average age of 47.43 years (21-72 years), and were all treated by modified piggyback orthotopic liver transplantation surgery. The details of the patient demographics are listed in Supplementary Table 1. The selection criteria were as published [17].
2.2. Animals. Six to eight weeks of C57BL/6J, BALB/c male mice were obtained from Guangzhou University of Chinese Medicine, China. YAP-HKO mice were constructed by crossing Albumin-Cre (Alb-Cre) mice and Yap flox/flox mice from the Model Animal Research Center of Nanjing University (Nanjing, China). CD11c-DTR mice were purchased from Jackson Laboratory (Farmington, CT, USA). All the animal experiments conducted in this study were approved by the animal ethics committee of the third affiliated hospital of Sun Yat-sen University.

2.3. Mouse Hepatic IRI Model. The 70\% liver ischemiareperfusion (I/R) injury model was constructed after $0.6 \%$ pentobarbital sodium $(100 \mu \mathrm{L} / 10 \mathrm{~g})$ was injected intraperitoneally [17]. In brief, the artery/portal vessel was clamped to the cephalad lobes for 90 minutes during ischemia, and reperfusion was performed by loosening the atraumatic vascular clamp. The sham operation group underwent the same operation except that blood vessels were not clamped. Animals received injections of EVs $(100 \mu \mathrm{g} / \mathrm{kg}$ b.w. in PBS) or PBS immediately before reperfusion.

2.4. Cells and Reagents. Human hepatic L02 cells from the Cell Bank of the Chinese Academy of Sciences in Shanghai were used in the in vitro experiments [18]. Antibodies against YAP1 (ab56701, Abcam), Alix (ab186429, Abcam), CD81 (D5O2Q, CST), TSG101 (ab125011, Abcam), $\beta$-actin (8H10D10, CST), calnexin (ab22595, Abcam), and CD47 (ab175388, Abcam) were used for western blot. When CD47 was blocked, two doses of $100 \mu \mathrm{g}$ (BE0270, BioXcell) were given intraperitoneally two days before IRI and the same day at IRI. The control group used the same dose of rat immunoglobulin G2a (IgG2a) isotype (BioXcell) [19].

2.5. EV Isolation and Analysis. EVs in mouse serum were isolated with an EV isolation kit (SmartSEC Mini EV Isolation System) and detected with an ELISA kit detecting CD81 exosome (EXOEL-CD81A-1) from System Biosciences.

L02 and primary hepatocytes (PMH) were cultured 48 hours at $37^{\circ} \mathrm{C}$ in serum-free DMEM. The supernatant was collected and centrifuged at $2,000 \mathrm{~g}$ for $10 \mathrm{~min}$ at $4^{\circ} \mathrm{C}$ to remove the cell debris [20]. Then, after filtering through $0.22 \mu \mathrm{m}$ filters, the filtrate was ultracentrifuged at $100,000 \mathrm{~g}$ for 120 minutes in a Beckman SW28Ti rotor. After the resuspension by $\mathrm{PBS}$, the pellets were ultracentrifuged again at $100,000 \mathrm{~g}$ for $120 \mathrm{~min}$. The final EV pellets were dissolved in PBS for further experiments [20].

To isolate CD $47^{+}$EVs, a total of $200 \mu \mathrm{g}$ PMH-EVs were mixed with nonblocking anti-CD47 antibody (REA170)FITC for $30 \mathrm{~min}$ at $4^{\circ} \mathrm{C}$. Then, incubated with anti-FITC magnetic beads (Miltenyi Biotec; $1 \mu \mathrm{L} / \mu \mathrm{g}$ EVs) overnight at $4^{\circ} \mathrm{C}$. CD $47^{-} \mathrm{EVs}$ and $\mathrm{CD} 47^{+} \mathrm{EVs}$ were separated by magnetic beads, and both supernatants were washed with PBS and pelleted by ultracentrifugation.

EVs were further analyzed by a Micro BCA Protein Assay Kit to determine protein concentrations (Thermo, \#23235). Particle diameters and amounts were observed by the NanoSight system (NS300, Malvern, Ranch Cucamonga, CA, 
USA). Further details of methods were described in supplementary materials (available here).

2.6. Real Time-PCR Analysis. Real-time PCR was performed using the following primer sequences [21]: YAP-forward: CCCAGACTACCTTGAAGCCA and YAP-reverse: CTTC CTGCAGACTTGGCATC; CYR61-forward: CTGCAGCAA AACTCAGCCCT and CACAGGGTCTGCCTTCTFAC and CYR61-reverse: CTTCCTGCAGACTTGGCATC; TRX1-forward: ATGGTGAAGCTGATCGAGAGC and TRX1-reverse: GGCATATTCAGTAATAGAGGC; HO-1forward: GCAGAGAATGCTGAGTTCATG and HO-1reverse: CACATCTATGTGGCCCTGGAGGAGG; GAPDH-forward: GCGGGAAATCGTGCGTGAC and GAPDH-reverse: CGTCATACTCCTGCTTGCTG.

2.7. Histopathology and Immunostaining. Tissues were fixed by $4 \%$ paraformaldehyde and embedded in paraffin, followed by slicing with a microtome and staining with hematoxylineosin. For the graft biopsy, the immunohistochemical staining results were assigned the mean score considering the product of the intensity of the stain and the percentage of positive cells. 0 is negative, 1 to 4 are mildly positive, 5 to 8 are moderately positive, and 9 to 12 are strong positive [22, 23]. Negatives are included in the low group, while weakly positive, moderately positive, and strong positives are included in the high group. Each section was independently assessed by two pathologists. Frozen liver tissue sections or cells were fixed, blocked according to standard procedures [19]. For immunofluorescence analysis, we used antibody YAP (2F12, Novus), RAB7 (ab137029, Abcam), EEA1 (610456, BD Biosciences) [24], LysoTracker ${ }^{\mathrm{TM}}$ Red DND-99 (ThermoFisher), Rhodamine Phalloidin (PHDR1, Cytoskeleton), Cy2-conjugated goat anti-rabbit IgG (111-225-144, Jackson ImmunoResearch), and Cy3-conjugated antimouse IgG (AP124C, EMD Millipore).

2.8. Plasmid Construction and Transfection. By short hairpin RNA inference, the silenced sh-YAP expression plasmids were purchased from Sigma, with the control group using the nontargeting shRNA expression plasmid (MISSION Plko.1-puro Empty Vector Control Plasmid DNA). The cells were transfected according to standard procedures [25].

2.9. Primary Hepatocyte Isolation. The liver was perfused with an immunoenzyme (10 units) to digest connective tissue [19]. Then, use Percoll gradient to purify primary hepatocytes.

2.10. Hepatic Lymphocyte Isolation. After the liver tissue was gently crushed, the lymphocytes were obtained using percoll gradient purification [19]. Then, the cell suspension was stained for viability analysis, and markers including CD45, CD47, CD172a, CD11c, I-A/I-E, TNF- $\alpha$, and IL-12 p40 (eBioscience, San Diego, CA).

2.11. $\mathrm{T}$ Cell Isolation. $\mathrm{CD}^{+}$and $\mathrm{CD} 8^{+} \mathrm{T}$ cells were isolated from the spleen of experiment mice by $\mathrm{CD} 4^{+}$and $\mathrm{CD} 8^{+} \mathrm{T}$ cell isolation kit II (Miltenyi Biotec, Bergisch Gladbach, Ger- many) and followed by labeling with CFSE (Invitrogen) according to the manufacturer's instructions [26].

2.12. DC Function Assay. Bone marrow monocytes were obtained from the cell suspension of mouse tibia and femur [27]. To generate bone marrow-derived DC (BMDCs), 10 $\mathrm{ng} / \mathrm{mL}$ recombinant mouse granulocyte-macrophage colony-stimulating factor (rmGM-CSF) and $1 \mathrm{ng} / \mathrm{mL}$ IL-4 $(\mathrm{R} \& \mathrm{D})$ were added in the medium (Figure S2C). BMDCs were collected after verifying purity (more than 95\% CD11c positive) and $1 \times 10^{6} / \mathrm{mL}$ cells were incubated under 1 $\mathrm{mg} / \mathrm{mL}$ LPS stimulation, and the experiment group was supplemented with $30 \mathrm{mg} / \mathrm{mL}$ EVs for $24 \mathrm{~h}$, with PBS supplemented as control. To evaluate the antigenpresenting ability of DCs, LPS-stimulated BMDCs were collected and cocultured with CFSE-labeled $\mathrm{CD}^{+}$and $\mathrm{CD}^{+} \mathrm{T}$ cells from BALB/c mouse at a ratio of $1: 10$ for 96 hours.

2.13. Cytokine ELISA. Both murine serum and culture supernatants from BMDCs were harvested for further cytokine analysis [28]. ELISA kits to measure IL-12 p40, TNF- $\alpha$, and IL-6 levels individually were purchased and used under the manufacturers' instructions (eBioscience, San Diego, CA).

2.14. Immunogold Electron Microscopy. For immune electron microscopy, we used anti-CD47 antibody (Novus-NBP244408 ) for primary staining and incubated overnight at $4^{\circ} \mathrm{C}$. Then, we applied $18 \mathrm{~nm}$ colloidal gold-conjugated goat anti-mouse IgG (115-215-166, Jackson ImmunoResearch) as the secondary antibodies. The following steps were performed according to previous reports [24].

2.15. Statistical Analyses. Student's $t$-test was used to analyze statistical comparisons between groups. The resulting $p$ value $<0.05$ is considered significant statistically.

\section{Results}

3.1. YAP Silencing during I/R Aggravates Liver Damage While Decreases Serum EV (sEV) Concentration. We first confirmed YAP expression level to be negatively correlated with hepatocellular damage in I/R-stressed human orthotopic liver transplantation (OLT) samples (Supplementary Fig. 1A-C, Supplementary Table 1) [4]. And during hepatic IRI in mice, hepatic YAP protein and YAP mRNA gradually increased over time (Figures 1(a) and 1(b)). Then, hepatocyte-specific YAP knockout (YAP-HKO) mice were constructed to study whether YAP knockout could aggregate hepatic dysfunction in hepatic IRI mice (Supplementary Fig. 1D-E), since the pan-tissue YAP knockout mice are lethal in a fetus. By these YAP-HKO mice with I/R at $24 \mathrm{~h}$, we observed severe hepatic damage by pathological analysis (Figure 1(c)) and measured their serum ALT levels (Figure 1(d)), while there were no obvious differences between YAP-HKO and wild-type (WT) mice under sham operation. Likewise, Suzuki's score indicated significantly increased hepatocellular damage by YAP silencing (Figure 1(e)). These data suggested that YAP silencing can exacerbate hepatic IRI. 


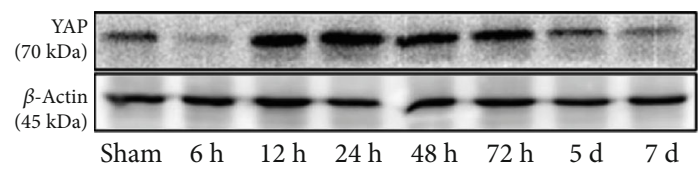

(a)

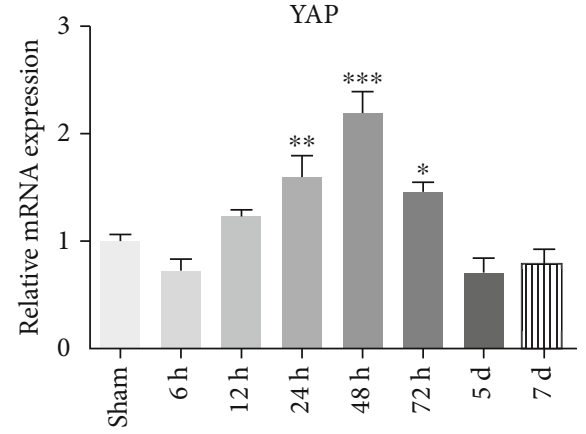

(b)
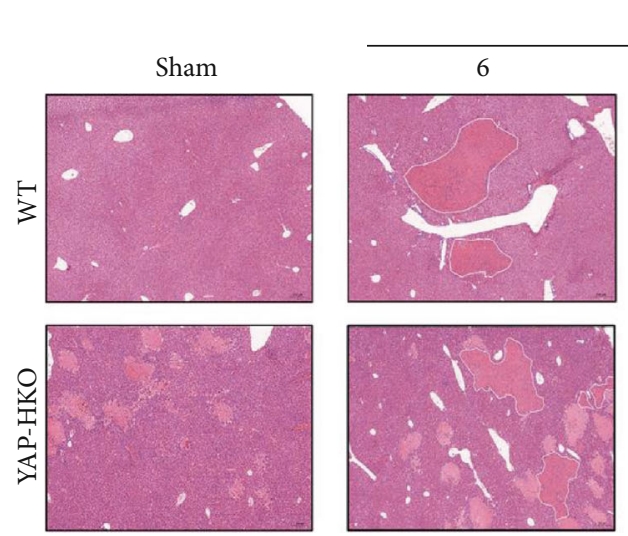

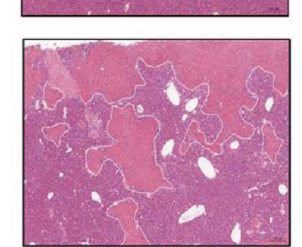

(c)
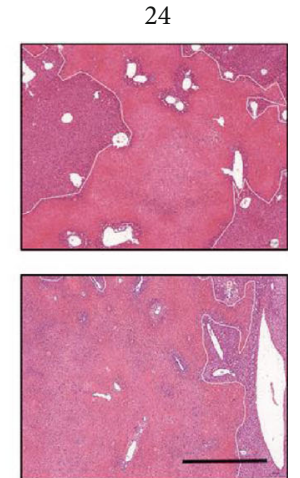

Serum EVs



(d)

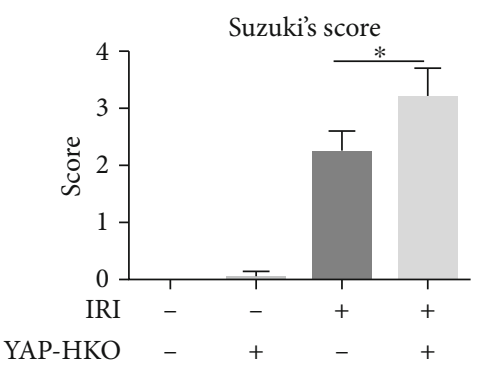

(e)



(f)

FIGURE 1: Silencing of YAP exacerbates liver injury and decreases sEVs' concentration during IRI. (a) Western blot analysis of hepatic YAP protein levels of IRI mice at different time points; (b) the mRNA levels of hepatic YAP from IRI mouse liver were detected by qPCR at different time points. (c) H\&E staining of ischemic livers in WT and YAP-HKO mice showed that the injury increased with reperfusion time and reached the peak at $24 \mathrm{~h}$ after reperfusion. Scale bar, $1 \mathrm{~mm}$. (d) Serum ALT levels of YAP-HKO mice were significantly higher than WT mice with I/R at $24 \mathrm{~h}\left(2059 \pm 214.2 \mathrm{U} / \mathrm{L}\right.$ vs. $\left.872.8 \pm 76.15 \mathrm{U} / \mathrm{L},{ }^{* *} p<0.01\right)$. (e) Suzuki's histological score of YAP-HKO mice was higher than WT mice with I/R at $24 \mathrm{~h}$ (score: $2.25 \pm 0.17$ vs. score: $3.2 \pm 0.25,{ }^{*} p<0.05$ ). (f) The concentrations of sEVs from WT and YAP-HKO groups at different time points after IRI, as measured by CD81 exosome ELISA kit. $n=6$ per group. ${ }^{* *} p<0.01$, ns: $p>0.05, t$-test.

Next, we isolated sEVs from WT mice and YAP-HKO mice after hepatic IRI with a serum EV isolation kit [29]. By CD81 exosome ELISA kit for quantification, we found that sEVs' concentration gradually increased after IRI and reached the peak in 24 hours (Figure 1(f)). This trend was in consistency with the YAP protein expression levels as shown in Figures 1(a) and 1(b). Silencing YAP in vivo decreased sEVs' concentration after IRI (Figure 1(f)). sEVs were verified by transmission electron microscopy (TEM) (Supplementary Fig. 2A). EV markers including CD81, ALIX, and TSG101 were expressed in sEV lysates, but the endoplasmic reticulum marker calnexin was not found in sEVs [30] (Supplementary Fig. 2B). Our in vivo observations indicate that sEVs' concentration is affected by YAP expression, and it is necessary to explore the mechanism and effect of YAP regulation on EV secretion. 
3.2. YAP Silencing Decreases the Formation of EVs in an FActin-Dependent Manner. To explore the effect of YAP on EV secretion from hepatocytes, we first found that lower YAP expression is correlated with smaller amount of secreted EVs from nanoparticle tracking analysis (NTA) and western blot analysis, by comparing EVs from WT and YAP knockdown (YAP-KD) L02 cells (the normal human hepatocyte line) during hypoxia/reoxygenation (Figure 2(a), Supplementary Fig. 2C, and Figure 2(b)). To elucidate the underlying mechanism of EV secretion in hepatocytes, we explored the role of the dynamic actin and branched actin networks in EV secretion under YAP regulation in vitro, which are on the surface of early endosome (EE). These networks are very important for membrane remodeling, which are critical for selective cargo sorting and EE shape control [7]. We found that most of the WT EE (marked by EEA1) were intensively covered by branched actin networks. In YAP-KD L02 cells, the amount of branched actin on EE decreased significantly (Figure 2(c)). Further analysis of YAP-KD L02 cells revealed that the recycling endosome (RE), which was formed by buddings from EE tubules, also decreased significantly as branched actin formation reduced, indicated by the decreased coposition of EE and F-actin on the plasma membrane (yellow). The RE is the main source of endosomal membrane formation. To study the role of F-actin in EV secretion under the regulation by YAP, we used cytochalasin $\mathrm{D}$ (CytoD) to induce F-actin depolymerization for 30 minutes. The depolymerized F-actin restored the inhibition of RE under YAP-KD condition, presenting as an increased level of copositioning of $\mathrm{EE}$ and F-actin on the plasma membrane (Figures 2(c) and 2(d)). At the same time, CytoD treatment also reversed the inhibitory effect of YAP-KD on EV secretion (Figure 2(e)). These results suggested that YAP might regulate the formation of EVs via $\mathrm{F}$-actin in hepatocytes.

3.3. YAP Expression Is Essential for Inhibiting the Fusion of $M V B$ and Lysosomes. Next, we evaluated whether YAP could induce EV secretion by inhibiting MVB and lysosome degradation. The dense reticulum of F-actin might form organelle traps in cells and slow down lysosome transport through an active F-actin anchoring mechanism [31]. We used late endosomes (LE, labeled with RAB7) to indicate MVE. With increased formation of F-actin patches in YAP-KD (Figure 2(c)), the fusion of lysosome (labeled by LysoTracker Red) with LE significantly increased (Figures 3(a) and 3(b)). When F-actin depolymerization was induced by CytoD, there was no significant difference in the colocalization of $\mathrm{LE}$ and lysosomal fluorescence between YAP-KD and WT L02 cells (Figure 3(b)). These results suggested that YAP induced EV secretion through F-actin by increasing membrane formation, as well as inhibiting the fusion of MVB and lysosomes.

3.4. CD47-Enriched EV Inhibits CD172 ${ }^{+}$DC Activation in a YAP-Dependent Manner. To determine the potential effect of YAP-induced EVs on hepatic IRI, we used quantitative mass spectrometry to detect EVs originated from WT L02 and YAP-KD L02 cells. 2883 trusted proteins were retrieved from the original data of mass spectrometry by Protein Pilot software (Supplementary Table 2, Figures 4(a) and 4(b)). Based on this result, we noticed CD47 was one of the target that highly expressed on hepatocyte-derived EVs in a YAPdependent manner and then verified its expression on primary mouse hepatocytes $(\mathrm{PMH})$ by western blotting (Supplementary Fig. 2D) and immunogold electron microscopy (Supplementary Fig. 2E). CD47 has been reported to exert its inhibitory effect by binding specifically to the surface receptor CD172 $\alpha$ of myeloid cells [32]. We examined whether CD47-enriched EVs target on the proinflammatory cells during hepatic IRI. By evaluating the inflammatory cytokines IL-12 p40 and TNF- $\alpha$ of CD $172^{+}$ cells in hepatic tissue with $I / R$ at $24 \mathrm{~h}$, we found that CD172 $\mathrm{a}^{+}$rather than CD172 $\mathrm{a}^{-}$cells are the main sources of IL-12 p40 and TNF- $\alpha$ (Figure 4(c)). Compared with the sham operation group, the ratio of CD172 $\mathrm{a}^{+} \mathrm{IL}-12 \mathrm{p} 40^{+}$or TNF- $\alpha^{+}$cells in the IRI group significantly increased (Figure 4(d)). Furthermore, we found that CD11c expression in hepatic tissues with $\mathrm{I} / \mathrm{R}$ was limited to the $\mathrm{CD}_{172 \mathrm{a}^{+}}$cell subset (Figure 4(e)). We next confirmed

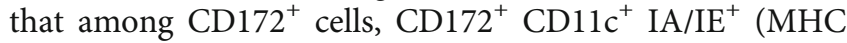
$\left.\mathrm{II}^{+}\right)$cells or $\mathrm{CD}_{172}{ }^{+}$DCs represented as the main producers of IL-12 p40 and TNF- $\alpha$ in hepatic IRI. These data suggested that the inflammatory cytokine production is restricted to CD172 $\mathrm{a}^{+}$DCs in hepatic IRI. As CD47 is located on the surface of EVs, we used CD47 neutralizing antibodies to neutralize EVs and found that neutralizing of CD47 from $\mathrm{PMH}-\mathrm{EV}$ s significantly antagonized the ability of these EVs to inhibit the secretion of inflammatory cytokines by bone-marrowderived dendritic cells (BMDCs) (Figure 4(f)). These results suggested that CD47-enriched EVs could suppress inflammatory responses of $\mathrm{CD} 172^{+}$DCs.

To evaluate the antigen-presenting function of DCs, we detected the proliferation of $\mathrm{CD}^{+}$and $\mathrm{CD}^{+} \mathrm{T}$ cells in vitro. In comparison to the YAP-HKO $\mathrm{PMH}$ treatment group, we found the proliferation rates were inhibited in BMDCs pretreated by a conditioned medium of WT PMH (Figures 5(a) and 5(b)). Meanwhile, by detecting IL-12 p40, we found LPS-induced-BMDC activation was significantly restrained by a PMH conditioned medium in a YAP-dependent manner (Figure 5(c)). These results implied that, in the absence of YAP, hepatocytes lose their ability to suppress BMDC activation in vitro.

To elucidate that YAP-dependent-DC function was regulated by hepatocyte-released EVs, we isolated EVs by ultracentrifugation from the supernatant of $\mathrm{PMH}$. We found EVs isolated from WT were more potent in inhibiting BMDCs than EVs from YAP-HKO PMH, as evaluated by IL-12 p40 secretion (Figure 5(c)). To distinguish between EVs and other nonmembrane extracellular particles [20], EVs were treated with detergent (Triton X-100) which can destroy the membrane structure and halt the functions of EVs. The particles after detergent treatment failed to inactivate BMDCs (Figure 5(c)). These results indicated that DC activation was inhibited by hepatocyte-released EVs in a YAP-dependent manner. 


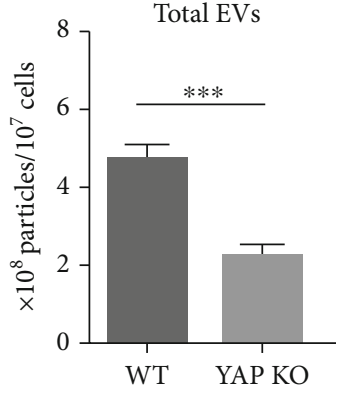

(a)

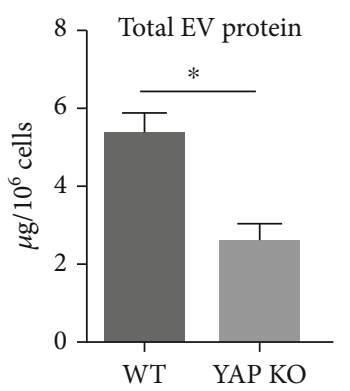

(b)

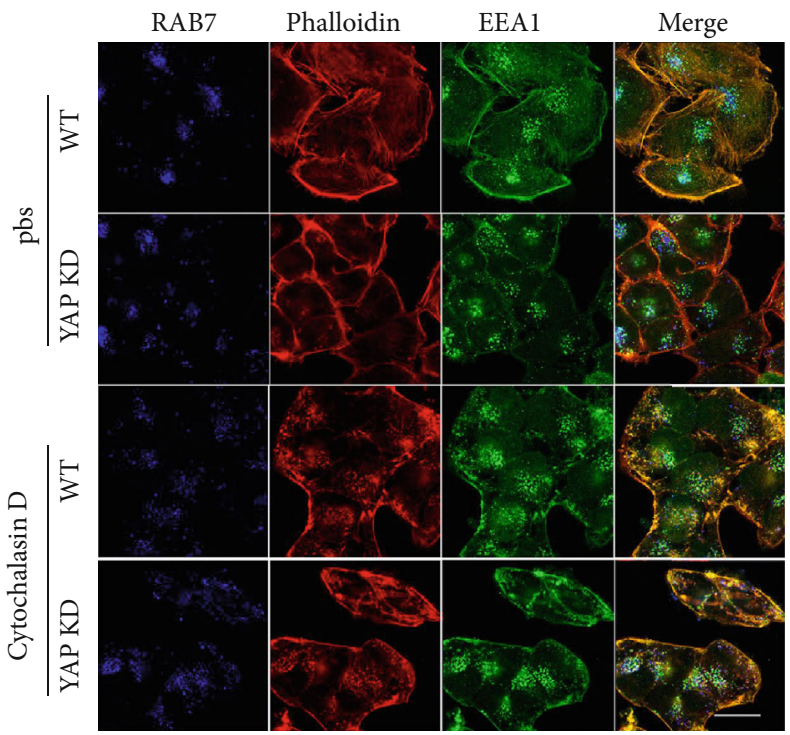

(c)

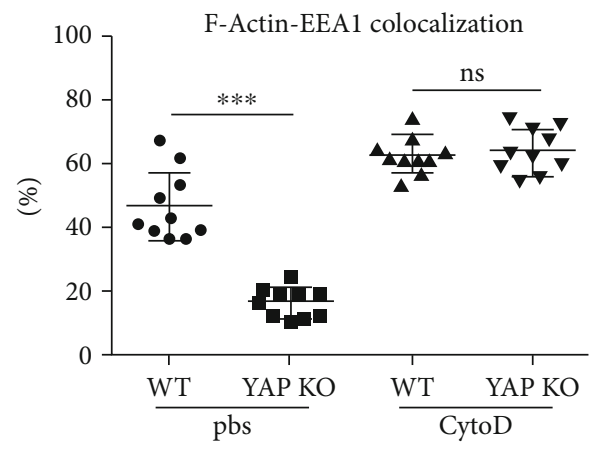

(d)

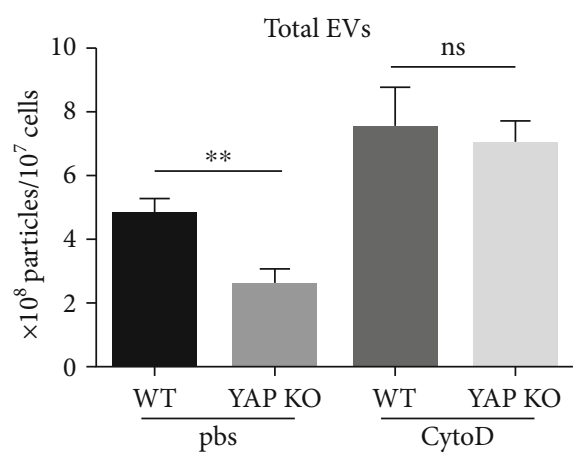

(e)

FIgURE 2: Hepatic YAP silence decreases EV formation. (a) EVs extracted from culture medium of WT or YAP-KD L02 cells were subjected to nanoparticle tracking analysis (NanoSight) for quantitative [20]. $n=3,{ }^{* * *} p<0.001, t$-test. (b) A BCA Protein Assay Kit was used to detect the protein concentration of EVs. $n=3 .{ }^{*} p<0.05$, $t$-test. (c) Rhodamine-labeled phalloidin staining (labeling membrane-associated F-actin, red), EEA1 staining (labeling early endosomes, green), and RAB7 staining (labeling late endosomes) for YAP-KD and WT L02 cells under either PBS or cytochalasin stimulation. Scale bar, $10 \mu \mathrm{m}$. (d) Statistical analysis of amounts of EEA1 that colocalized (orange) with F-actin, shown as percentages of the total EEA1, $n=10,{ }^{* * *} p<0.001$, ns: $p>0.05, t$-test. (e) EVs extracted from a culture medium of equal amount of WT or YAP-KD L02 cells with or without CytoD treatment were subjected to NanoSight for quantification. $n=3$. ${ }^{* *} p<0.01$, ns: $p>0.05, t$-test.

3.5. CD $47^{+}$EVs Could Alleviate Hepatic IRI as a Therapeutic Strategy. To investigate whether CD $47^{+}$EVs have the potential to treat hepatic IRI, we first isolated CD $47^{+}$EVs and CD47 ${ }^{-}$EVs using nonblocking anti-CD47 Ab (REA170)FITC and anti-FITC magnetic beads. We then verified the purified $\mathrm{CD}_{4} 7^{+} \mathrm{EVs}$ contain CD47 and EVs' label proteins
TSG101 and Alix (Figures 6(a) and 6(b)). Next, we injected $\mathrm{CD}_{4} 7^{+}$EVs into WT mice to determine whether CD $47^{+}$ EVs have any adverse effect, and we found there was no damage to normal mice without IRI. While in the IRI model, less hepatic damage was observed in the group treated by $\mathrm{CD} 47^{+}$ EVs (Figures 6(c) and 6(d)). We found the expression of 


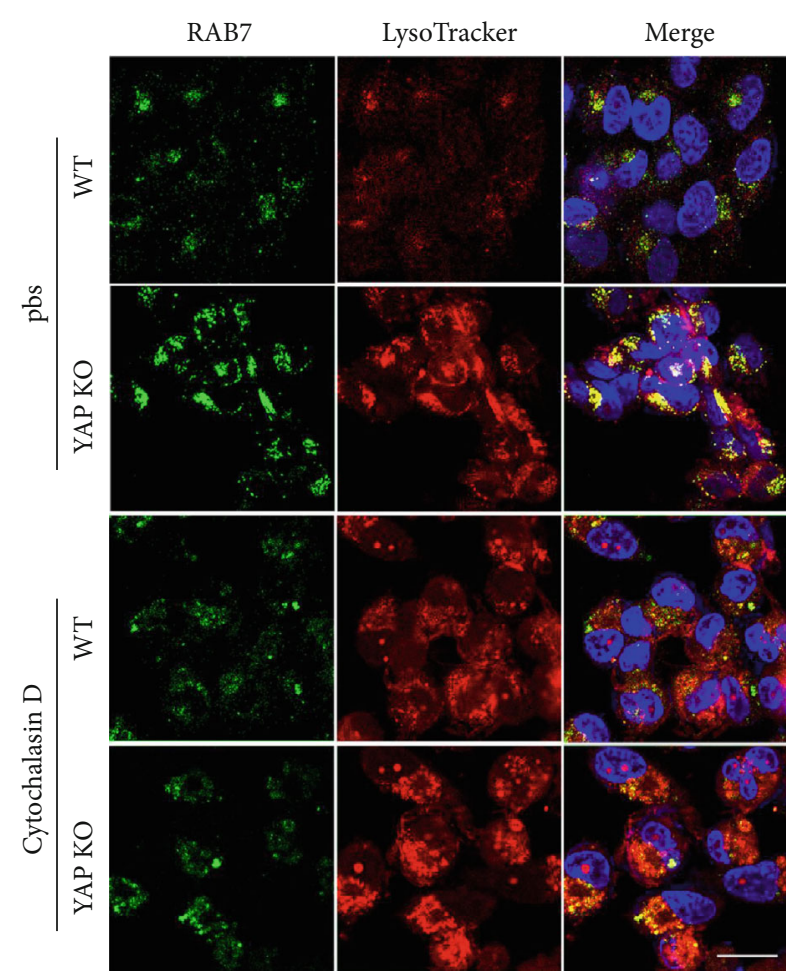

(a)

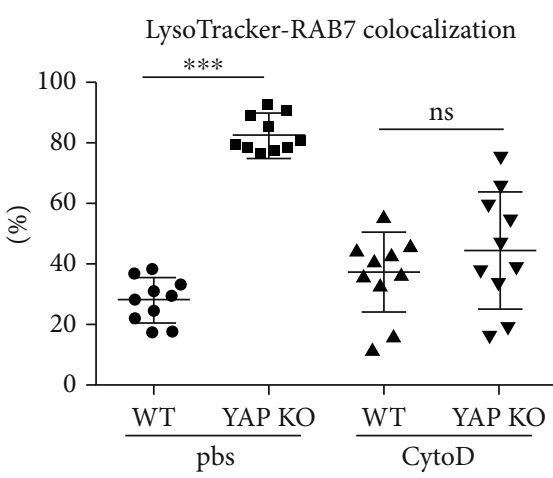

(b)

FIGURE 3: YAP expression is required for the fusion of MVB and lysosomes. (a) Representative image of the fusion of lysosome (labeled by LysoTracker staining, red) with late endosomes (labeled by RAB7 staining, green) significantly increased in YAP-KD compared to WT L02 cells with or without CytoD treatment. Scale bar, $10 \mu \mathrm{m}$. (b) Statistical analysis of the amounts of RAB7 ${ }^{+}$granules colocalize (orange) with lysosome, $n=10,{ }^{* * *} p<0.001$, ns: $p>0.05$, $t$-test.

antioxidative genes increased including thioredoxin-1 (Trx1) and heme oxygenase-1 (HO-1) after $\mathrm{CD} 47^{+} \mathrm{EV}$ treatment. Further, we examined the expression of Cyr61, which has been recognized as a key chemokine controlling liver injury [5]. The mRNA levels of hepatic Cyr61 were reduced after CD $47^{+}$EV treatment (Supplementary Fig. 2F). As CD $47 m A b$ could reverse the protective effects of $\mathrm{CD} 47^{+} \mathrm{EVs}$, the protection of these EVs on hepatic IRI is possibly mediated by CD47 (Figures 6(c) and 6(d)). In addition, there was no significant amelioration in hepatic damage after I/R in CD11cDTR mice injected with DT after $\mathrm{CD} 47^{+} \mathrm{EV}$ treatment (Figures 6(c) and 6(d)), suggesting that CD $47^{+}$EVs alleviate hepatic IRI at least partially depending on DCs. Similarly, $\mathrm{CD}_{4} 7^{+} \mathrm{EV}$ injection reduced the serum levels of IL- 6 and TNF- $\alpha$, while this effect was reversed by CD $47 \mathrm{mAb}$ (Figure 6(e)). These results showed that CD $47^{+}$EVs may be a potential therapeutic strategy for the treatment of hepatic IRI.

Taken together, our data showed that YAP regulates the secretion of hepatocyte-derived EVs, which can inhibit DC activation through its surface CD47 and contribute to liver protection during hepatic IRI.

\section{Discussion}

In this study, we identified a novel mechanism that YAP activation affects the release of hepatic EVs, which regulates DC activation through CD47/CD172a axis. CD47-enriched EVs could be a novel therapeutic strategy for treating hepatic IRI by targeting on CD172a ${ }^{+}$DCs.

The participation of YAP in IRI has been reported in different studies with controversial effects $[4,5]$. In our study, hepatic YAP protein and mRNA were induced in a timedependent manner in hepatic IRI, demonstrating the requisite role of YAP. This notion was strongly supported by human OLT samples. Further, we reported that conditional knockout YAP in hepatocytes led to severe hepatic damage with IRI, by crossing Albumin-Cre (Alb-Cre) mice and Yapflox/flox mice [34]. These mice displayed increased serum ALT levels and pathological hepatic changes, indicating YAP silencing played a pivotal role in aggregating hepatic IRI. Then, via serum EV isolation kit, we found in the serum of YAP-HKO mice, the amount of sEVs decreased after IRI. Thus, we further investigated how YAP knockout affects sEV secretion and exacerbates hepatic injuries.

Actually, it has been found that IRI can induce the secretion of EVs [29], but how YAP knockout in IRI decreased EV secretion remains unclear. EVs are formed through direct germination of plasma membrane or germination of endomembrane structure after fusing with late endosomes (LE) or multivesicular body (MVB) and are secreted based on the process that fuses with plasma membrane [35]. During this process, the branched actin network is known to play a key role in endosomal trafficking and EV secretion [36]. YAP can regulate the actin-binding activity of AMOT family members by competing with F-actin for binding to 


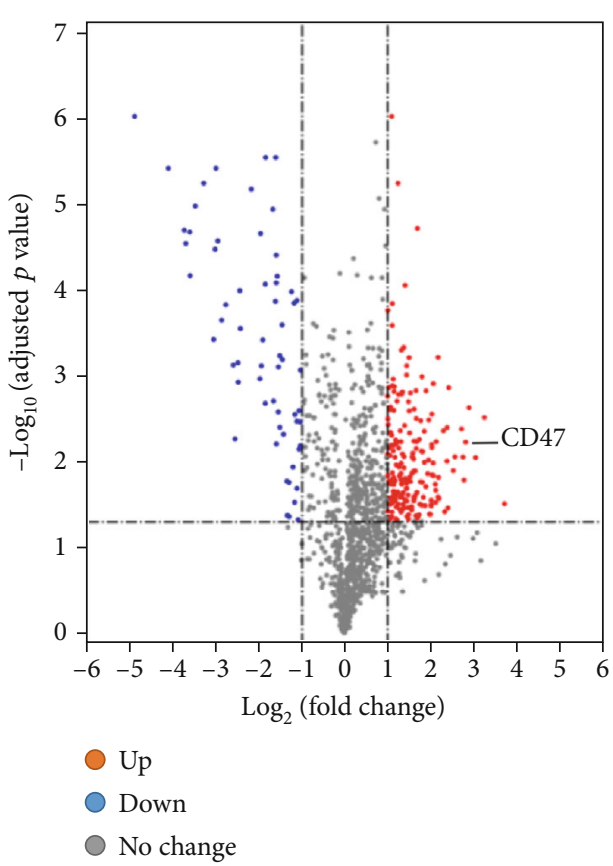

(a)


(c)

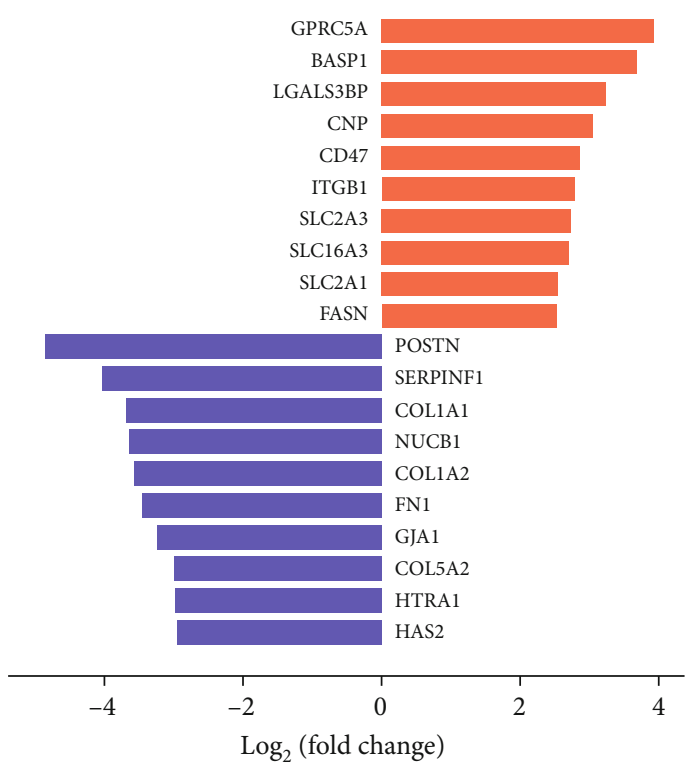

(b)
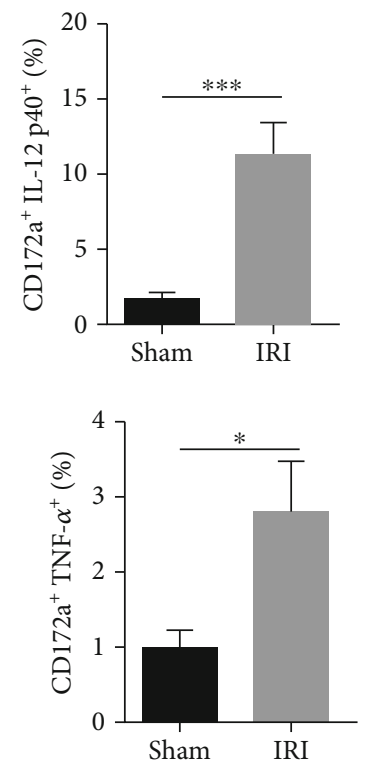

(d)

FIgURE 4: Continued. 


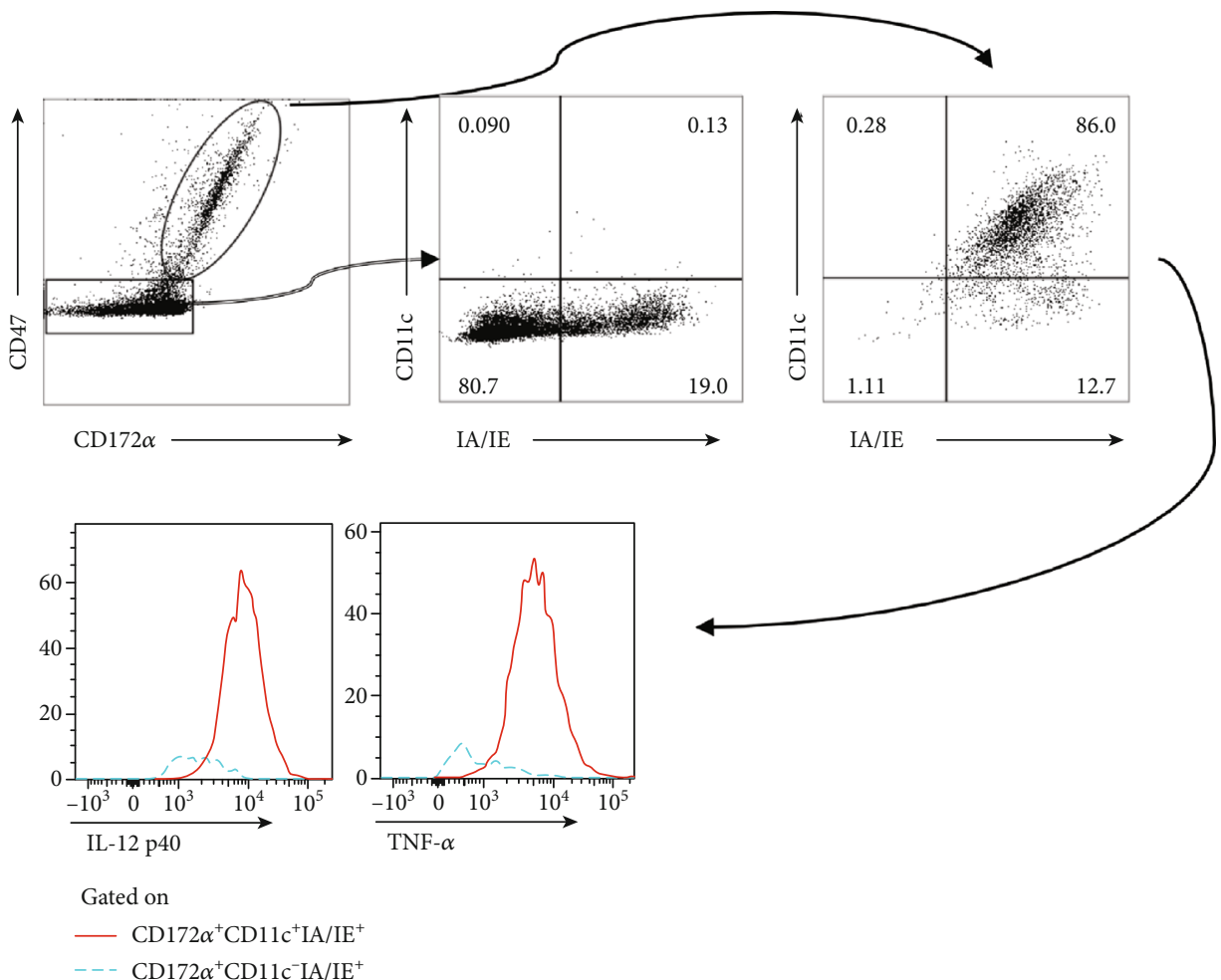

(e)
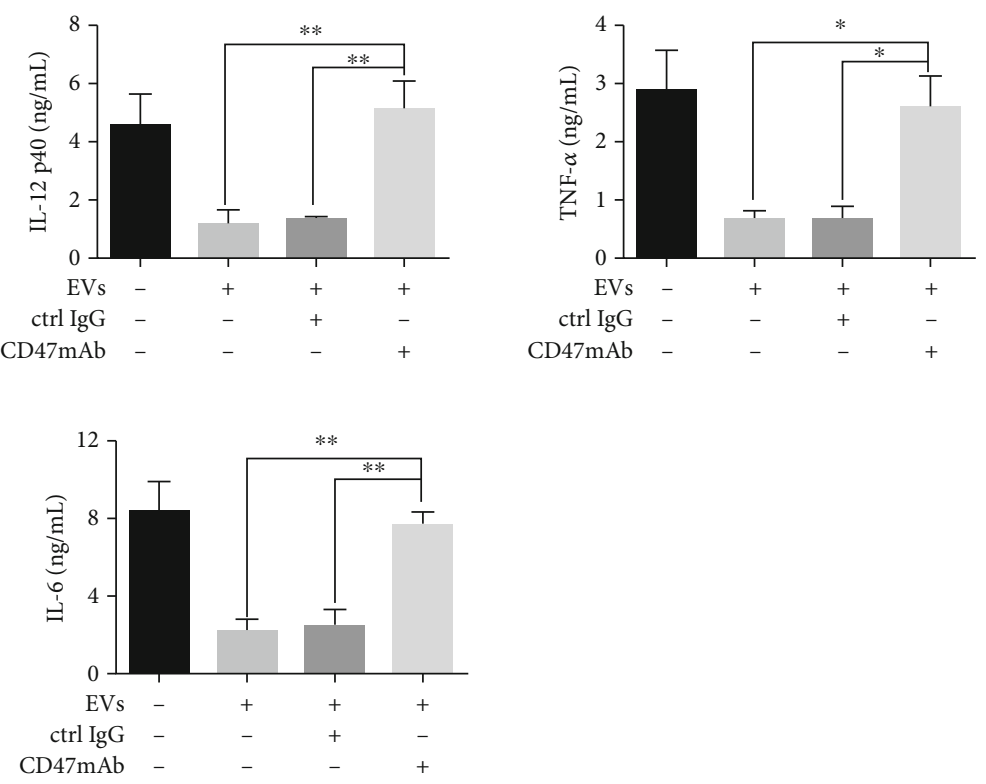

(f)

FIGURE 4: CD47-enriched EVs inhibit CD172a ${ }^{+}$DC activation. (a) Volcano map presenting the 62 downregulated and 277 upregulated proteins in EVs originated from WT L02 and YAP-KD L02 cells by mass spectrometry, in which CD47 were among the upregulated proteins. (b) Bar chart showing the top 10 upregulated and downregulated proteins with CD47 ranked top four. Protein expression (Ex) was converted to $\log 2(\mathrm{Ex})$, and the scale represented relative expression [20]. (c) Representative flow cytometry images of CD45 ${ }^{+}$cells stained by CD172a combined with IL-12 p40 or TNF- $\alpha$ in sham or IRI mouse models. (d) The proportion of CD172a ${ }^{+}$IL-12 p40 ${ }^{+}$or CD172 $\mathrm{a}^{+}$TNF- $\alpha^{+}$cells was shown. Bars represent the subset amounts of CD172a ${ }^{+}$IL- 12 p $40^{+}$or CD172a ${ }^{+}$TNF- $\alpha^{+}$cells $($mean \pm s.d., $n=$ 4 mice). (e) CD47 and CD172 $\alpha$ expressions were analyzed on hepatic immune cell populations. CD172 $\alpha^{+}$cells were further subdivided according to CD11c and IA/IE expressions. Intracellular expression of cytokines (IL-12 p40 and TNF- $\alpha$ ) was examined on CD172 $\alpha^{+}$

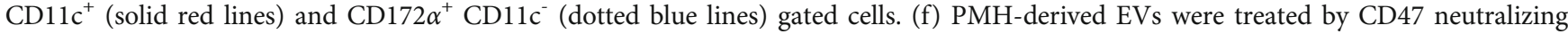
antibody or control IgG. qPCR analysis showed a significant increase in inflammatory cytokines including IL-12 p40, TNF- $\alpha$, and IL-6 after CD47 neutralization. $n=6,{ }^{*} p<0.05 ;{ }^{* *} p<0.01 ;{ }^{* * *} p<0.001$. 


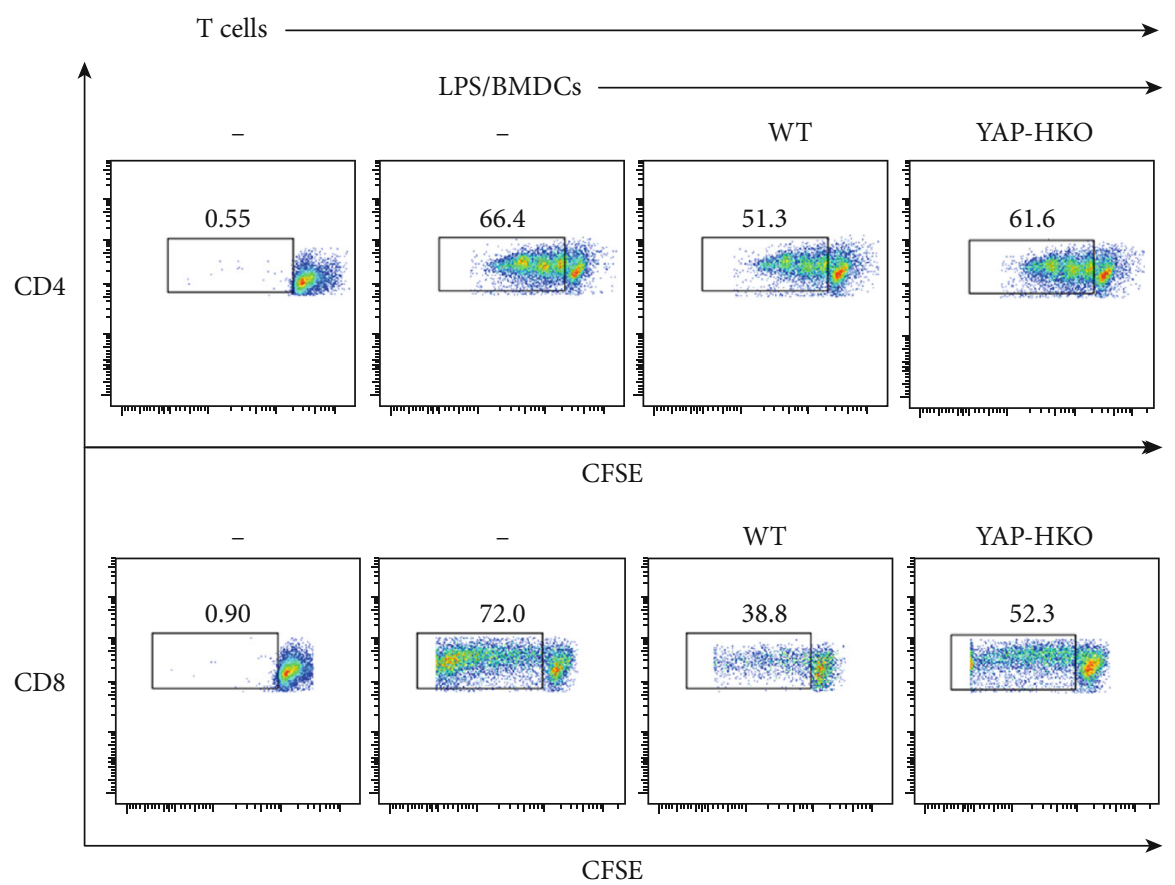

(a)
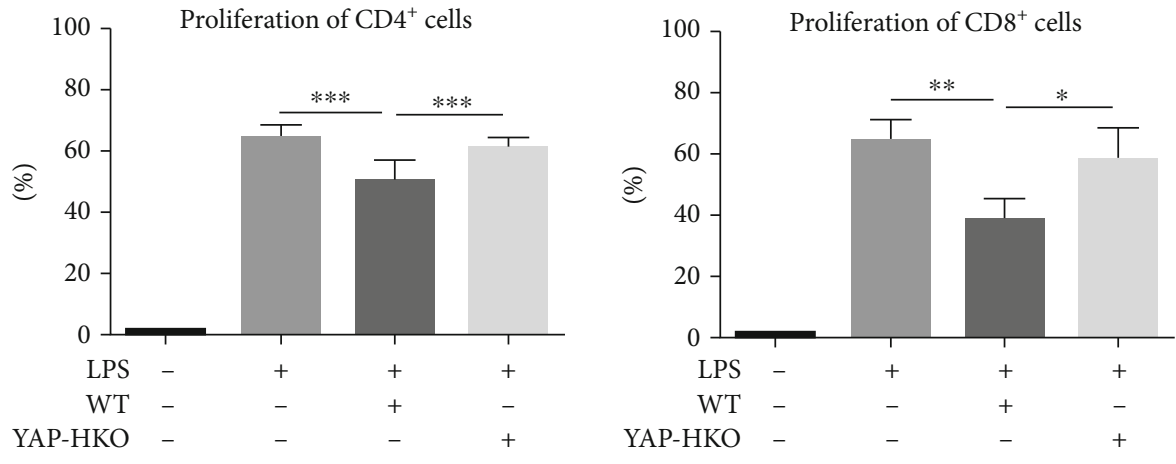

(b)

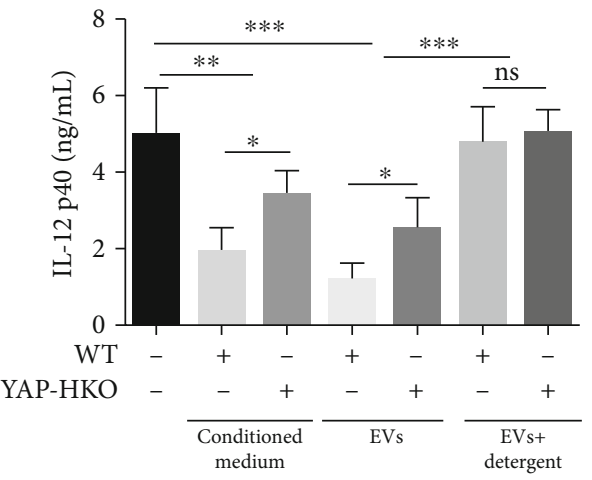

(c)

Figure 5: EVs released from hepatocytes inhibit DC activation in a YAP-dependent manner. (a) Primary BMDCs were treated by a conditioned medium from WT or YAP-HKO PMH and were stimulated with LPS. BMDCs were then cocultured with CD4 ${ }^{+}$and $\mathrm{CD} 8^{+} \mathrm{T}$ cells isolated from BALB/c mice at the ratio of $1: 10$. The proliferation of CD4 $4^{+}$and $\mathrm{CD} 8^{+} \mathrm{T}$ cells was measured by CFSE-MLR. (b) The extent of $\mathrm{T}$ cell proliferation in (a) was analyzed. (c) BMDCs were treated with a conditioned medium or EVs from WT or YAP-HKO PMH culture supernatants, and then, the IL-12 p40 level in the culture medium was determined by ELISA. ${ }^{*} p<0.05 ;{ }^{* *} p<0.01 ;{ }^{* * *} p<$ 0.001. $n=4$. 


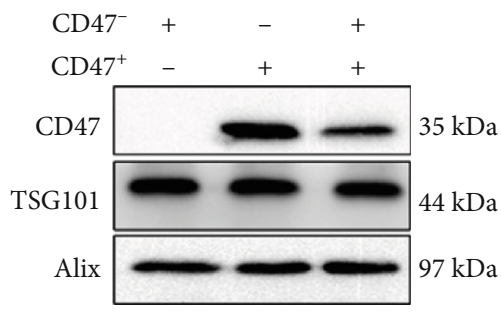

(a)

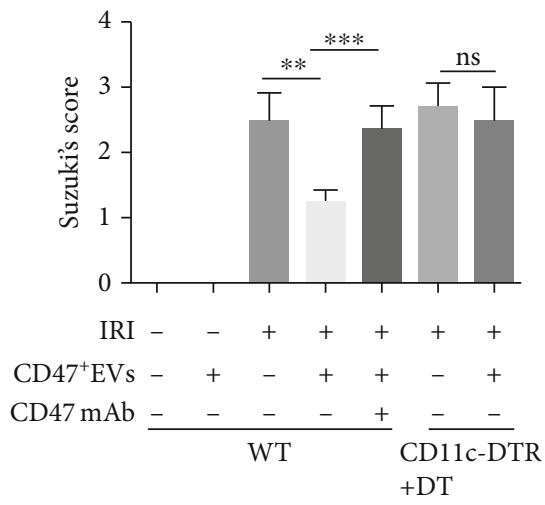

(c)

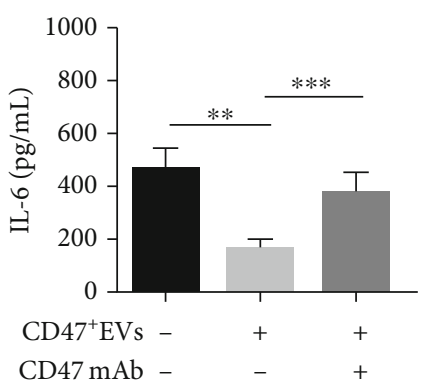

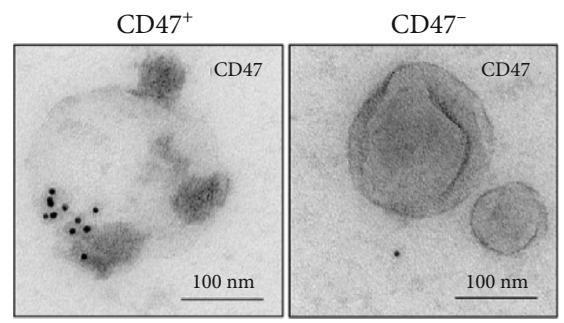

(b)

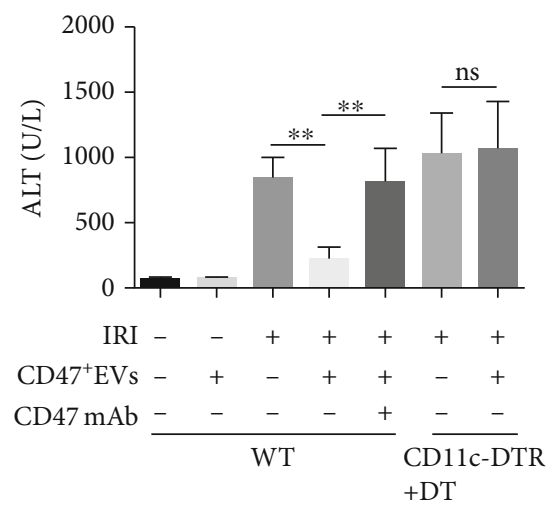

(d)

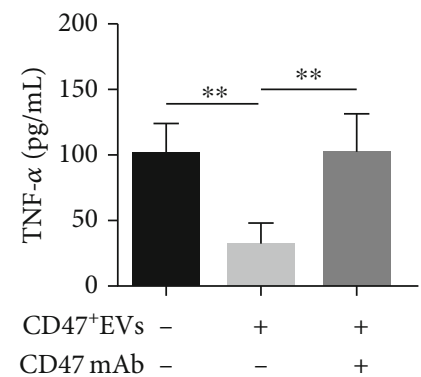

(e)

FIgure 6: $\mathrm{CD}_{4} 7^{+}$EVs alleviate hepatic IRI in mouse models. (a) $\mathrm{CD} 47^{+}$EVs and CD47 EVs were isolated by nonblocking anti-CD47 Ab (REA170)-FITC and anti-FITC magnetic beads. Alix, TSG101, and CD47 in CD47 ${ }^{+}, \mathrm{CD}^{-} 7^{-}$, and total PMH-EVs were checked by western blot. (b) Immunogold electron microscopy images showed the immunoreactivity for CD47 on CD47 ${ }^{+}$and CD47 EVs. (c) CD11c-DTR were treated with DT (4 mg/kg b.w.) and/or plus with CD $47^{+} \mathrm{EVs}(100 \mu \mathrm{g} / \mathrm{kg}$ b.w.) on 2 and 0 days before IRI model construction. The severity of hepatic IRI was evaluated by Suzuki's grading [33]. (d) Hepatocellular function was assessed by serum ALT level (U/L). (e) ELISA analysis showed decreased concentrations of IL- 6 and TNF- $\alpha$ after CD $47^{+}$EVs supply without CD47mAb treatment during IRI. $n$ $=6,{ }^{*} p<0.05 ;{ }^{* *} p<0.01 ;{ }^{* * *} p<0.001$.

AMOT130 [6]. It is possible that YAP may regulate EV secretion by regulating F-actin. In our study, we investigated the mechanism involved in endosomal trafficking, which involves the Rab family of small GTPases, lysosomes, and remodeled actin [37]. We found that YAP knockdown by lentivirus shRNA significantly reduced EV release, suggesting that YAP participated in EV secretion. By comparing WT and YAP-KD hepatocytes, we found that YAP-KD decreased the formation of branching actin. By limiting the fusion of intraluminal vesicles and cell membranes, it is conducive to the development of RE and further decreases the occurrence of EV membrane [24,38]. RE is closely related to the generation of EVs [39]. Both dynamic and branched actin could reduce the fusion of LE and lysosome, thereby increasing the secretory MVE as EVs. Therefore, we are the first to report that YAP from hepatocytes regulate EV secretion through F-actin in hepatic IRI. But our works focused on the role of EVs released from hepatocytes; further studies are needed to explore the effect of EVs from other nonparenchymal cells, such as Kupffer cells, liver sinusoidal endothelial cells, and immune cells. Besides, autophagy has been reported to promote the fusion of MVB and autophagy [40], and YAP is well known to regulate autophagy flux by promoting autolysosome degradation [41]. Whether YAP regulated EV secretion through autophagy requires further study.

Then, to investigate the potential effect and mechanism of EVs in hepatic IRI, we applied mass spectrometry to explore the essential elements. Among the top factors, we found that CD47 exists on the surface of EV membrane. 

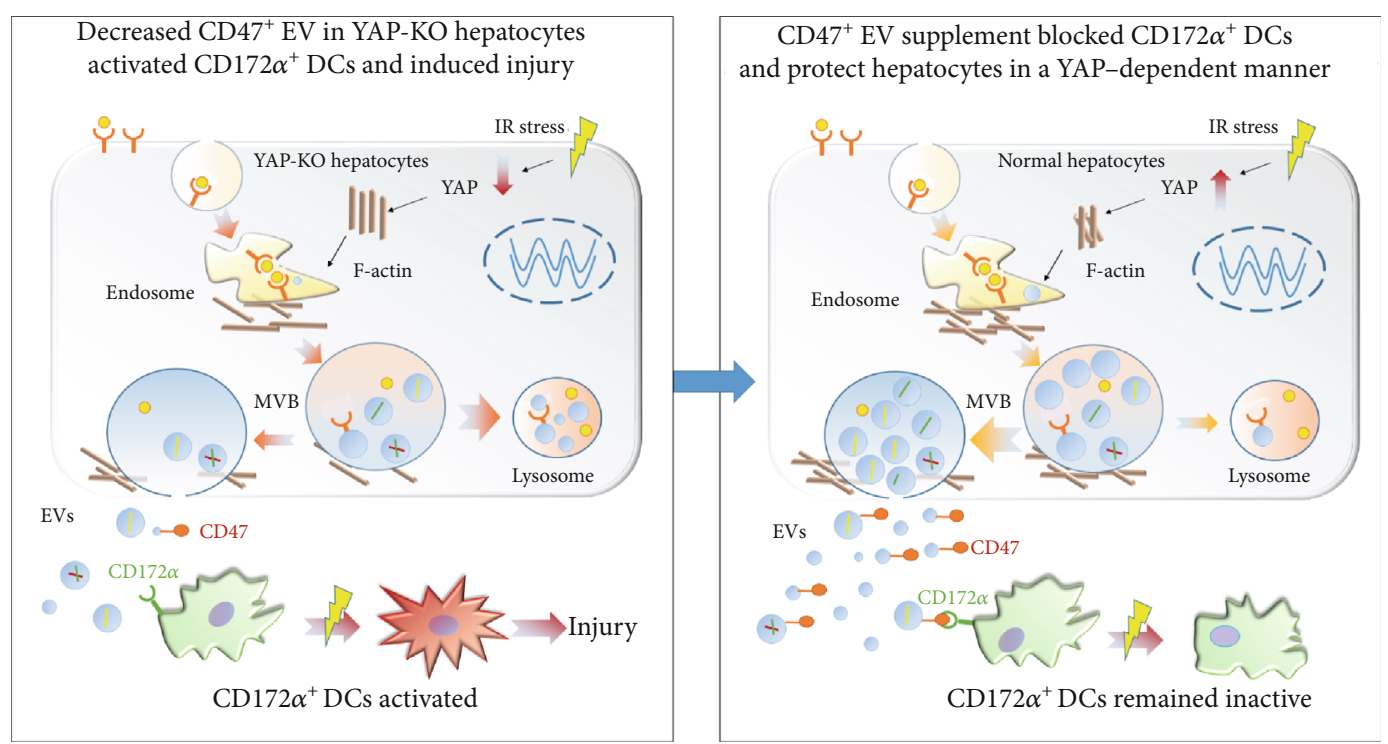

FIGURE 7: Mechanism diagram for the immunosuppression effect of YAP/F-actin/EV-CD47 axis on DCs in hepatic IRI. IR stress stimulated YAP expression in normal hepatocytes and promoted the remodeling of F-actin to form an endosomal trafficking network, which is critical for EV formation. These EVs were rich in CD47, which could bind to CD172a receptor on DCs. In normal conditions, most of the MVB would form into EVs with a little few into lysosome. When YAP expression was knocked down, however, F-actin failed to gather around and form a network report; thus, the amount of EVs decreased with more vesicles engulfed by lysosome. The amount of CD47 was scarce, and $\mathrm{CD}_{172^{+}}$DCs were activated and led to more severe damage. Therefore, EVs lack of CD $47^{+}$due to YAP knockout in hepatocytes, activated DCs, and aggregated hepatic IRI, while YAP activation induced by hepatic IRI promoted the secretion of CD47-enriched EVs by remodeling of cytoskeleton F-actin, and $\mathrm{CD}_{4} 7^{+}$EVs induced the immunosuppressive DCs and protected the liver from IRI. CD47 ${ }^{+} \mathrm{EV}$ supplement could be a novel therapeutic strategy for hepatic IRI treatment.

CD47 is considered to be the self-recognition marker that specially binds CD172 $\alpha$, which profoundly inhibits the secretion of inflammatory cytokines. The latest tumor research uses CD47 to modify nanoparticles, which reduces the clearance rate of nanoparticles by macrophages [42]. Studies have shown that CD47 not only takes part in autoimmune diseases and tumors but also plays a key role in IRI-related diseases in kidneys and hearts $[43,44]$. And CD172 $\alpha^{+}$DCs, as target cells of CD47, are the main cells secreting proinflammatory cytokines in hepatic IRI [15]. CD47mAb blockade confirmed that EV-associated CD47 take the immunosuppressive effect on DCs and reduced hepatic IRI. Under normal conditions, DCs remain in an inactivated state. Under inflammation stress, DCs are exposed to foreign antigens, microorganisms, and inflammatory cytokines resulting in activation and then subsequently initiating immune response [28]. Our results highlighted the regulatory role of YAP in the regulation of DC function during hepatic IRI. In this study, we observed that hepatocyte culture medium obviously inhibited LPSinduced IL-12 p40 production and antigen-presenting ability of BMDCs in a YAP-dependent manner. We suspected that under stress, YAP induced EV secretion from hepatocytes, which have the immunosuppressive effect on DCs. Interestingly, we treated EVs with detergent, which destructed the membrane structures of EVs, reversing the inhibitory effect of EVs on DCs. This suggested that YAP induced the secretion of EVs to exert an immunosuppressive effect on DCs. In addition, we found that CD47+ EVs can inhibit DC activation and inflammatory responses, which can explain their protective effects on IRI. Together, these data suggest that
CD47-enriched-EVs can inhibit DC activation and decrease subsequent hepatic damage.

Innate immunity is the dominant ingredient of liver IRI, among which dendritic cells, macrophage, and neutrophils are pivotal participants [45]. In comparison with neutrophils and macrophages, DCs are the dominant local immune cells surveilling and maintaining immune homeostasis with less complex phenotype switches [46]. Moreover, DC has been determined as the main contributors in hepatic IRI, affecting the development of adaptive immunity by regulating the initial response and amplifying the innate immune response [11]. The role of other immune cells such as macrophages or neutrophils in hepatic IRI needs further investigation.

EVs have been proved to be ideal cargos for drug delivery, which showed better stability, easier preservation, and more precise targeting in comparison to traditional cell therapy or RNA drugs [47]. The advantages of EVs make them attractive as the potential candidates for treating many diseases. The specific structure of phospholipid bilayer enables EVs to protect their contents from in vivo degradation and the disturbances from inflammatory microenvironment [48]. Besides, EVs could be modified and produced in a standard process. Therefore, CD47-enriched EVs could be engineered by integrating EVs with CD47 loading, which could be manufactured at a large scale independent of the cell origin, which could be the future direction of precise drug development.

In summary, our study reported a novel regulatory mechanism between EVs and DCs depending on YAP activation in hepatic IRI (Figure 7). Specifically, YAP knockout 
decreased the release of EVs in hepatocytes by affecting Factin, which reduced membrane formation and promoted MVB to fuse into lysosomes. The YAP-deficient EVs are lack of CD47, which failed to inactivate CD172 $\alpha^{+}$DCs and lead to sustained IRI injury. CD47-enriched EV treatment blocked CD $172 \alpha^{+}$DCs and protected IRI mice from hepatic injury. Our findings suggested that the secretion of $\mathrm{CD} 47^{+} \mathrm{EVs}$ depending on YAP activation played a protective role during hepatic IRI, and CD47-enriched EVs could be a novel therapeutic strategy for hepatic IRI treatment.

\section{Abbreviations}

$\begin{array}{ll}\text { ALT: } & \text { Alanine aminotransferase } \\ \text { AMOT: } & \text { Angiomotin } \\ \text { BM: } & \text { Bone marrow } \\ \text { CytoD: } & \text { Cytochalasin D } \\ \text { DCs: } & \text { Dendritic cells } \\ \text { DT: } & \text { Diphtheria toxin } \\ \text { EVs: } & \text { Extracellular vesicles } \\ \text { HO-1: } & \text { Heme oxygenase-1 } \\ \text { I/R: } & \text { Ischemia-reperfusion } \\ \text { IRI: } & \text { Ischemia-reperfusion injury } \\ \text { LPS: } & \text { Lipopolysaccharide } \\ \text { MVB: } & \text { Multivesicular body } \\ \text { MLR: } & \text { Mixed leukocyte reaction } \\ \text { NanoSight: } & \text { Nanoparticle tracking analysis } \\ \text { OLT: } & \text { Orthotopic liver transplantation } \\ \text { PMH: } & \text { Primary mouse hepatocyte } \\ \text { SIRPa: } & \text { Signal-regulated protein alpha } \\ \text { Trxl: } & \text { Thioredoxin-1 } \\ \text { WT: } & \text { Wild-type } \\ \text { YAP: } & \text { Yes-associated protein } \\ \text { YAP-HKO: } & \text { Hepatocyte-specific YAP knockout. } \\ & \end{array}$

\section{Data Availability}

All data relevant to the study are included in the article or uploaded as supporting information.

\section{Ethical Approval}

This study was approved by the ethics institutions of the Third Affiliated Hospital of Sun Yat-sen University, Guangdong Province, China. In detail, the experiments relating to clinical data were approved by the Clinical Ethics Review Board, while all animal procedures were approved by the animal experimental ethics committee.

\section{Consent}

All participants agreed on the results in this study and signed informed consent for publication.

\section{Conflicts of Interest}

No conflict of interest was declared by all authors.

\section{Authors' Contributions}

Zenan Yuan and Hua Li designed the experiments and analyzed the data. Linsen Ye and Xiao Feng performed the surgical procedure. Tian Zhou assisted in analyzing the data and writing the manuscript. Yi Zhou, Shuguang Zhu, and Changchang Jia helped in acquiring data. Haibo Li, Dongbo Qiu, Kun Li, Wei Liu, and Hui Tang assisted in analyzing data. Guoying Wang and Qi Zhang contributed to collecting samples. Li Hua, Guihua Chen, and Yang Yang supervised the study. All authors approved the final manuscript. Zenan Yuan, Linsen Ye, Xiao Feng, and Tian Zhou contributed equally to this work.

\section{Acknowledgments}

This study was supported by the National Key R\&D Plan (2017YFA0104304), the China Postdoctoral Science Foundation (2020M672980), the National Natural Science Foundation of China (81702393, 81770648, 81802897, 81802897, and 81900886), the National 13th Five-Year Science and Technology Plan Major Projects of China (2017ZX10203205-006-001), the Basic and Applied Basic Research Foundation of Guangdong Province (2021A1515010726), the Key Scientific and Technological Projects of Guangdong Province (2017A030311034), the Guangdong Natural Science Foundation (2017A030310373), the Science and Technology Planning Project of Guangdong Province (2017B030314027, 2017B020209004, 20169013, and 2017A020215178), the Science and Technology Planning Project of Guangzhou (201607010024 and 201604020001), and the Sun Yat-sen University Young Teacher Training Project (17ykpy47 and 17ykzd27).

\section{Supplementary Materials}

Supplementary Figure 1: (A) representative images of YAP expression in liver graft by immunohistochemistry (left panel), 37 patients were in the YAP high-expression group and 32 patients were in the YAP low-expression group; representative histology of liver by H\&E staining (right panel) from indicated groups. Magnification $\times 200$. (B) Suzuki's histological grading of the YAP high-expression group was significantly lower than the low-expression group (score: $1.60 \pm 0.08$ vs. score: $\left.2.37 \pm 0.13,{ }^{* * *} p<0.001\right)$. (C) The peak serum ALT within 7 days after transplantation of the highexpression group were significantly lower than the lowexpression group $(448.4 \pm 22.78 \mathrm{U} / \mathrm{L}$ vs. $653.9 \pm 36.44 \mathrm{U} / \mathrm{L}$, $\left.{ }^{* * *} p<0.001\right)$. (D) The schematic diagram of design strategy of YAP-HKO mice, and the plasmid map of the targeting vector used in YAP-HKO mice. YAP-HKO mice were constructed by crossing Albumin-Cre (Alb-Cre) mice and Yapflox/flox mice. (E) YAP protein levels of the liver from two independent WT and two independent YAP-HKO mice were measured by western blot. Supplementary Figure 2: (A) EVs isolated from mouse serum were visually confirmed by transmission electron microscopy, and (B) subjected to western blot analysis with antibodies to the indicated proteins. (C) WT L02 cells secrete more EVs. EVs isolated from culture 
supernatants of WT or YAP knockout (KO) L02 cells were subjected to nanoparticle tracking analysis (NanoSight) to quantify the number and size distribution. (D) A total of $35 \mu \mathrm{g}$ of EVs isolated from culture supernatants of WT or YAP-HKO PMH were analyzed by western blot using the indicated antibodies. Data are representative of three independent experiments. (E) Immunogold electron microscopy analysis depicts the presence of CD47 on the surface of PMH-derived EVs. (F) Quantitative RT-PCR-assisted detection of Trx1, HO-1, and Cyr61 after CD47 ${ }^{+}$EV treatment during IRI. Supplementary Table 1: demographics, perioperative situation, and laboratory results of liver transplantation surgery patients. Supplementary Table 2: the 62 downregulated and 277 upregulated proteins in EVs originated from WT L02 and YAP-KD L02 cells by mass spectrometry. (Supplementary Materials)

\section{References}

[1] M. A. Zimmerman, I. Kam, H. Eltzschig, and A. Grenz, "Biological implications of extracellular adenosine in hepatic ischemia and reperfusion injury," American Journal of Transplantation, vol. 13, no. 10, pp. 2524-2529, 2013.

[2] D. Papadopoulos, T. Siempis, E. Theodorakou, and G. Tsoulfas, "Hepatic ischemia and reperfusion injury and trauma: current concepts," Arch Trauma Res., vol. 2, no. 2, pp. 63-70, 2013.

[3] J. P. Duffy, K. Kao, C. Y. Ko et al., "Long-term patient outcome and quality of life after liver transplantation: analysis of 20year survivors," Annals of Surgery, vol. 252, no. 4, pp. 652$661,2010$.

[4] Y. Liu, T. Lu, C. Zhang et al., "Activation of YAP attenuates hepatic damage and fibrosis in liver ischemia- reperfusion injury," Journal of Hepatology, vol. 71, no. 4, pp. 719-730, 2019.

[5] M. Mooring, B. H. Fowl, S. Z. C. Lum et al., "Hepatocyte stress increases expression of yes-associated protein and transcriptional coactivator with PDZ-binding motif in hepatocytes to promote parenchymal inflammation and fibrosis," Hepatology, vol. 71, no. 5, pp. 1813-1830, 2020, Epub 2019/09/11.

[6] S. W. Chan, C. J. Lim, F. S. Guo, I. Tan, T. Leung, and W. J. Hong, "Actin-binding and cell proliferation activities of angiomotin family members are regulated by Hippo pathwaymediated phosphorylation*," Journal of Biological Chemistry, vol. 288, no. 52, pp. 37296-37307, 2013.

[7] K. G. Campellone and M. D. Welch, "A nucleator arms race: cellular control of actin assembly," Nature Reviews. Molecular Cell Biology, vol. 11, no. 4, pp. 237-251, 2010.

[8] J. Wolfers, A. Lozier, G. Raposo et al., "Tumor-derived exosomes are a source of shared tumor rejection antigens for CTL cross-priming," Nature Medicine, vol. 7, no. 3, pp. 297303, 2001.

[9] H. Peinado, M. Alečković, S. Lavotshkin et al., "Melanoma exosomes educate bone marrow progenitor cells toward a pro- metastatic phenotype through MET," Nature Medicine, vol. 18, no. 6, pp. 883-891, 2012.

[10] Y. Zhai, X. D. Shen, R. O'Connell et al., "Cutting edge: TLR4 activation mediates liver ischemia/reperfusion inflammatory response via IFN regulatory factor 3-dependent MyD88independent pathway," Journal of Immunology, vol. 173, no. 12, pp. 7115-7119, 2004.
[11] T. L. Sumpter, M. Abe, D. Tokita, and A. W. Thomson, "Dendritic cells, the liver, and transplantation," Hepatology, vol. 46, no. 6, pp. 2021-2031, 2007.

[12] R. M. Steinman, "The dendritic cell system and its role in immunogenicity," Annual Review of Immunology, vol. 9, no. 1, pp. 271-296, 1991.

[13] T. Nakao, Y. Ono, H. Dai et al., "DNAX activating protein of $12 \mathrm{kDa}$ /triggering receptor expressed on myeloid cells 2 expression by mouse and human liver dendritic cells: functional implications and regulation of liver ischemiareperfusion injury," Hepatology, vol. 70, no. 2, pp. 696-710, 2019.

[14] D. J. van Rees, K. Szilagyi, T. W. Kuijpers, H. L. Matlung, and T. K. van den Berg, "Immunoreceptors on neutrophils," Seminars in Immunology, vol. 28, no. 2, pp. 94-108, 2016, Epub 2016/03/16.

[15] A. N. Barclay and T. K. Van den Berg, "The interaction between signal regulatory protein alpha $(\operatorname{SIRP} \alpha)$ and $\mathrm{CD} 47$ : structure, function, and therapeutic target," Annual Review of Immunology, vol. 32, no. 1, pp. 25-50, 2014.

[16] B. Buttari, E. Profumo, B. Cuccu et al., "Erythrocytes from patients with carotid atherosclerosis fail to control dendritic cell maturation," International Journal of Cardiology, vol. 155, no. 3, pp. 484-486, 2012.

[17] Y. Li, D. Y. Ruan, C. C. Jia et al., "Aging aggravates hepatic ischemia-reperfusion injury in mice by impairing mitophagy with the involvement of the EIF $2 \alpha$-parkin pathway," Aging (Albany NY), vol. 10, no. 8, pp. 1902-1920, 2018, Epub 2018/08/10.

[18] X. Li, S. Yi, Y. Deng et al., "MiR-124 protects human hepatic L02 cells from $\mathrm{H}_{2} \mathrm{O}_{2}$-induced apoptosis by targeting Rab38 gene," Biochemical and Biophysical Research Communications, vol. 450, no. 1, pp. 148-153, 2014, Epub 2014/05/31.

[19] V. L. Kolachala, S. Palle, M. Shen, A. Feng, D. Shayakhmetov, and N. A. Gupta, "Loss of L-selectin-guided CD8(+), but not CD4(+), cells protects against ischemia reperfusion injury in a steatotic liver," Hepatology, vol. 66, no. 4, pp. 1258-1274, 2017.

[20] T. Moroishi, T. Hayashi, W. W. Pan et al., "The hippo pathway kinases LATS1/2 suppress cancer immunity," Cell, vol. 167, no. 6, pp. 1525-1539.e17, 2016, e17.

[21] C. Shi, Y. Cai, Y. Li et al., "Yap promotes hepatocellular carcinoma metastasis and mobilization via governing cofilin/Factin/lamellipodium axis by regulation of JNK/Bnip3/SERCA/CaMKII pathways," Redox Biology, vol. 14, pp. 59-71, 2018, Epub 2017/09/05.

[22] C. L. Da, Y. Xin, J. Zhao, and X. D. Luo, "Significance and relationship between yes-associated protein and survivin expression in gastric carcinoma and precancerous lesions," World Journal of Gastroenterology, vol. 15, no. 32, pp. 4055-4061, 2009.

[23] Y. Xunyi, Y. Zhentao, J. Dandan, and L. Funian, "Clinicopathological significance of PTPN12 expression in human breast cancer," Revista Brasileira de Pesquisas Médicas e Biológicas, vol. 45, no. 12, pp. 1334-1340, 2012, Epub 2012/10/10.

[24] S. Yoon, A. Kovalenko, K. Bogdanov, and D. Wallach, "MLKL, the protein that mediates necroptosis, also regulates endosomal trafficking and extracellular vesicle generation," Immunity, vol. 47, no. 1, pp. 51-65.e7, 2017, e7.

[25] Z. Yuan, J. Zhang, Y. Huang et al., "NRF2 overexpression in mesenchymal stem cells induces stem-cell marker expression 
and enhances osteoblastic differentiation," Biochemical and Biophysical Research Communications, vol. 491, no. 1, pp. 228-235, 2017.

[26] H. Ji, X. D. Shen, Y. Zhang et al., "Activation of cyclic adenosine monophosphate-dependent protein kinase a signaling prevents liver ischemia/reperfusion injury in mice," Liver transplantation : official publication of the American Association for the Study of Liver Diseases and the International Liver Transplantation Society., vol. 18, no. 6, pp. 659-670, 2012, Epub 2012/02/01.

[27] L. Jiang, Y. Shen, D. Guo et al., "EpCAM-dependent extracellular vesicles from intestinal epithelial cells maintain intestinal tract immune balance," Nature Communications, vol. 7, no. 1, p. 13045, 2016, Epub 2016/10/11.

[28] B. Ke, X. D. Shen, N. Kamo et al., “ $\beta$-Catenin regulates innate and adaptive immunity in mouse liver ischemia-reperfusion injury," Hepatology, vol. 57, no. 3, pp. 1203-1214, 2013, Epub 2012/10/20.

[29] M.-q. Yang, Q. Du, J. Goswami et al., "Interferon regulatory factor 1-Rab27a regulated extracellular vesicles promote liver ischemia/reperfusion injury," Hepatology, vol. 67, pp. 10561070, 2018.

[30] Y. Aoki, R. Manzano, Y. Lee et al., "C9orf72 and RAB7L1 regulate vesicle trafficking in amyotrophic lateral sclerosis and frontotemporal dementia," Brain : a journal of neurology., vol. 140, no. 4, pp. 887-897, 2017, Epub 2017/03/24.

[31] B. van Bommel, A. Konietzny, O. Kobler, J. Bar, and M. Mikhaylova, "F-Actin patches associated with glutamatergic synapses control positioning of dendritic lysosomes," The EMBO Journal, vol. 38, no. 15, article e101183, 2019.

[32] S. Pengam, J. Durand, C. Usal et al., "SIRP $\alpha / C D 47$ axis controls the maintenance of transplant tolerance sustained by myeloid-derived suppressor cells," American Journal of Transplantation, vol. 19, no. 12, pp. 3263-3275, 2019.

[33] Q. Zhu, C. Li, K. Wang et al., "Phosphatase and tensin homo$\log -\beta$-catenin signaling modulates regulatory $\mathrm{T}$ cells and inflammatory responses in mouse liver ischemia/reperfusion injury," Liver transplantation : official publication of the American Association for the Study of Liver Diseases and the International Liver Transplantation Society., vol. 23, no. 6, pp. 813825, 2017, Epub 2017/02/06.

[34] S. V. Iverson, K. M. Comstock, J. A. Kundert, and E. E. Schmidt, "Contributions of new hepatocyte lineages to liver growth, maintenance, and regeneration in mice," Hepatology, vol. 54, no. 2, pp. 655-663, 2011.

[35] C. C. Scott, F. Vacca, and J. Gruenberg, "Endosome maturation, transport and functions," Seminars in Cell \& Developmental Biology, vol. 31, pp. 2-10, 2014.

[36] M. A. Puthenveedu, B. Lauffer, P. Temkin et al., "Sequencedependent sorting of recycling proteins by actin-stabilized endosomal microdomains," Cell, vol. 143, no. 5, pp. 761-773, 2010.

[37] H. Bai, Q. Zhu, A. Surcel et al., "Yes-associated protein impacts adherens junction assembly through regulating actin cytoskeleton organization," American Journal of Physiology. Gastrointestinal and Liver Physiology, vol. 311, no. 3, pp. G396-G411, 2016.

[38] A. Akhter, K. Caution, A. Abu Khweek et al., "Caspase-11 promotes the fusion of phagosomes harboring pathogenic bacteria with lysosomes by modulating actin polymerization," Immunity, vol. 37, no. 1, pp. 35-47, 2012.
[39] S. Lemoinne, D. Thabut, C. Housset et al., "The emerging roles of microvesicles in liver diseases," Nature Reviews. Gastroenterology \& Hepatology, vol. 11, no. 6, pp. 350-361, 2014.

[40] C. M. Fader, D. Sanchez, M. Furlan, and M. I. Colombo, "Induction of autophagy promotes fusion of multivesicular bodies with autophagic vacuoles in k562 cells," Traffic, vol. 9, no. 2, pp. 230-250, 2008.

[41] Q. Song, B. Mao, J. Cheng et al., "YAP enhances autophagic flux to promote breast cancer cell survival in response to nutrient deprivation," PLoS One, vol. 10, no. 3, article e0120790, 2015.

[42] Z. Belhadj, B. He, H. L. Deng et al., "A combined "eat me/don't eat me" strategy based on extracellular vesicles for anticancer nanomedicine," Journal of Extracellular Vesicles, vol. 9, no. 1, p. 1806444, 2020.

[43] X. Wang, M. Xu, J. Jia et al., "CD47 blockade reduces ischemia/reperfusion injury in donation after cardiac death rat kidney transplantation," American Journal of Transplantation, vol. 18, no. 4, pp. 843-854, 2018.

[44] N. M. Rogers, M. Yao, E. M. Novelli, A. W. Thomson, D. D. Roberts, and J. S. Isenberg, "Activated CD47 regulates multiple vascular and stress responses: implications for acute kidney injury and its management," American Journal of Physiology. Renal Physiology, vol. 303, no. 8, pp. F1117-F1125, 2012.

[45] Y. Zhai, R. W. Busuttil, and J. W. Kupiec-Weglinski, "Liver ischemia and reperfusion injury: new insights into mechanisms of innate-adaptive immune-mediated tissue inflammation," American Journal of Transplantation, vol. 11, no. 8, pp. 1563-1569, 2011.

[46] T. Kaisho and S. Akira, "Dendritic-cell function in toll-like receptor- and MyD88-knockout mice," Trends in Immunology, vol. 22, no. 2, pp. 78-83, 2001.

[47] J. P. Armstrong, M. N. Holme, and M. M. Stevens, "Re-engineering extracellular vesicles as smart nanoscale therapeutics," ACS Nano, vol. 11, no. 1, pp. 69-83, 2017, Epub 2017/01/10.

[48] L. Han, J. Xu, Q. Xu, B. Zhang, E. W. Lam, and Y. Sun, "Extracellular vesicles in the tumor microenvironment: therapeutic resistance, clinical biomarkers, and targeting strategies," Medicinal Research Reviews, vol. 37, no. 6, pp. 1318-1349, 2017, Epub 2017/06/07. 